\title{
THE GUARANÍ LAND - CHECKLIST OF HORNWORTS (ANTHOCEROTOPHYTA) AND LIVERWORTS (MARCHANTIOPHYTA) OF PARAGUAY ${ }^{1}$
}

\author{
LARS SÖDERStröm, ANDERs Hagborg, MATt vON KonRAT \& ANA SÉNECA
}

\begin{abstract}
The first ever liverwort and hornwort checklist is provided for Paraguay. Despite the high level of biological diversity in Paraguay, there remain very few intensive collecting efforts for liverworts and hornworts in the region since the late 1800's. We report 2 hornwort taxa and 71 liverwort taxa. The list is based on almost 100 literature references, including monographs, regional studies, and molecular investigations. Given the dramatic loss of habitats in Paraguay coupled with high species diversity in other organisms, further collecting of liverworts and hornworts is critical. There is also the potential to use historical records with contemporary collections to investigate the impact of habitat loss in the area.
\end{abstract}

Key words: Marchantiophyta, Anthocerotophyta, biodiversity, taxa

Lars Söderström, Department of Biology, Norwegian University of Science and Technology, N-7491 Trondheim, Norway; email: lars.soderstrom@ntnu.no

Anders Hagborg \& Matt von Konrat, Science and Education, The Field Museum, Chicago, Illinois 60605-2496, U.S.A.; e-mail: hagborg@pobox.com,mkonrat@fieldmuseum.org

Ana Séneca, FCUP, Department of Biology, Rua Campo Alegre, Ed. FC4, S/N, 4169-007 Porto, Portugal and Norwegian University of Science and Technology, N-7491 Trondheim, Norway; email: aseneca@fc.up.pt, aseneca@bio.ntnu.no

\section{INTRODUCTION}

The Republic of Paraguay (hereafter referred to as Paraguay) is situated almost in the center of South America where it is bordered to the south and southwest by Argentina, to the east and northeast by Brazil, and to the northwest by Bolivia. It's main population refer to themselves as Guaraní, which also is the name of one of the official languages and of the currency. Although Paraguay is mostly lowland (the highest point, Cerro Peró, is at $842 \mathrm{~m}$ ), eastern Paraguay includes one of the worlds 35 biodiversity hotspots, the Atlantic forest. The Atlantic forest is the second largest rainforest of the American continent, once stretching almost continuously along the Brazilian coast and extending inland in the south and into eastern Paraguay and northeastern Argentina (Tabarelli et al.

\footnotetext{
1 We dedicate this checklist to our friend and colleague Tamás Pócs. He has probably never been in Paraguay. If he had, the number of known species would have been much higher.
}

2005). In the last three decades, alarming deforestation rates and the low percentage of Atlantic forest left by the 2000s call for immediate actions to halt the trends of forest loss (Huang et al. 2009). Hence, the Atlantic forest in South America has been identified as one of the top priority ecosystems for global biological conservation (Myers 1988; Olson \& Dinerstein 2002). While the Atlantic forest in eastern Paraguay only accounts for a small portion of the geographic area of the Atlantic Forest ecoregion, it has an disproportionately high level of species diversity (Huang et al. 2009). The number of vascular plant species in eastern Paraguay was estimated at the order of 10,000 (Zardini 1993), while the number of invertebrate species in Paraguay was around 100,000 (Catterson \& Fragano 2004).

The only systematic collection of liverworts from Paraguay dates back to the late 1800's, and was carried out by Benjamin Balansa who spent 
considerable time there. His first stay there was from 1873 to 1877 , the second was from 1878 to 1884. He spent most of his time in Asunción and on his estate in the Paraguarí province (Fourier 2005). Spruce (1888) reported a list of 28 liverworts based on Balansa's material and he later (Spruce 1890) described the collection publishing 14 new species. We here present the first ever checklist of 73 liverworts and hornworts for Paraguay in an effort to further promote bryological research in the region, and provide a foundation for revisionary studies and floristic research. As a comparison, the moss checklist for Paraguay (O'Shea \& Price 2008) includes 240 taxa. This checklist fills a major gap in our knowledge of terrestrial plants from this region. The significance of checklists is summarized by Söderström et al. (2008), including outlining the utility of checklists as powerful and important tools, and their applicability to taxonomy, systematics, and conservation. Given the recent massive loss of forest habitats in Paraguay, the checklist may serve for as baseline inquiry with land management implications with further collecting efforts.

\section{VEGETATION}

Paraguay is dominated by three main vegetation types (Olson et al. 2001; Fig. 1). The eastern part of the country is covered by Alto Paraná Atlantic forests and is the most humid part of the country. It is also from here that most species are known (Fig. 2). The central part of the country is covered with Humid Chaco, which consists of xenophile forests mixed with palm savannas. The western part is covered by Dry Chaco which consists of grasslands and thorn forests.

\section{METHODS AND PRESENTATION}

The present checklist is based on a compilation of all the published records of species and infraspecific taxa of liverworts and hornworts from Paraguay that we are aware of. The Early Land Plant Today (ELPT) database (von Konrat et al. 2010) was an important source. The format of the present checklist follows that of the database. Accordingly, confidence levels are provided for each taxon, as follows (Söderström et al. 2010;

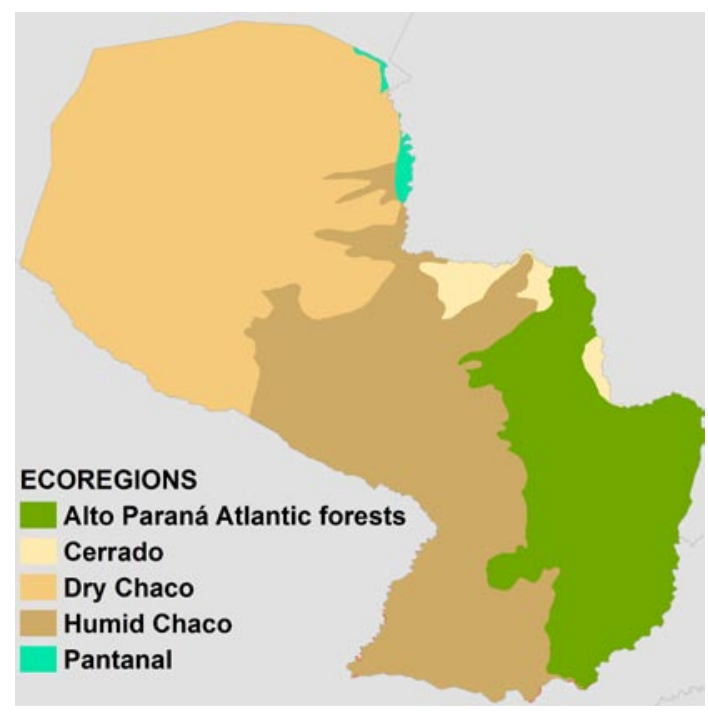

Fig. 1. The main ecoregions of Paraguay.

von Konrat et al. 2010): * - serious doubts about the value of the taxon, ${ }^{* *}$ - probably a good taxon, but not studied critically in the framework of a recent revision or monograph, ${ }^{* * *}$ - good taxon as currently understood, studied in the framework of a recent revision or monograph.

No attempt has been made to examine the specimens of the published records and verify the identifications. However, as in the recent checklists in the same series, a distinction is made between primary references (providing details on specimens and/or exact localities) and secondary ones (lacking details). Primary references are in small capitals and secondary ones in normal font.

Nomenclature follows the latest taxonomic opinions as much as possible and author abbreviations are according to the International Plant Names Index (http://www.ipni.org/index.html). Taxa doubtfully present in Paraguay and synonyms are listed separately. The list of synonyms only contains names used for Paraguay.

The following abbreviations are used for the provinces: AG (Alto Paraguay, $1 \mathrm{sp}$.), AN (Alto Paraná, $21 \mathrm{sp}$.), AM (Amambay, 4 sp.), AS (Asunción, 9 sp.), CC Concepción, CG (Caaguazú, 7 sp.), CZ (Caazapá, 3 sp.), CE (Central, 1 sp.), CR (Cordillera, 8 sp.), GU (Guairá, 5 sp.), IT (Itapúa, 3 sp.), PA (Paraguarí, 17 sp.), PH (Presidente Hayes, 1 sp.) and PAR (lack of precise locality information in the cited references). We do not have any reports of hornworts or liverworts for the remaining six provinces. There are a few localities that 


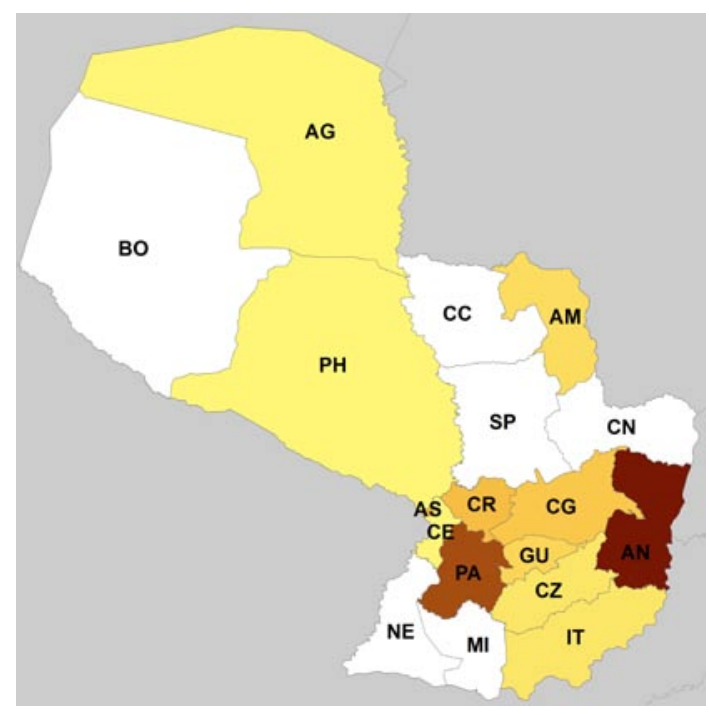

Fig. 2. Number of species recorded in each province in Paraguay. The darker the more species (max. 21 species). There are no known reports from provinces in white. AG - Alto Paraguay, AN - Alto Paraná, AM - Amambay, AS - Asunción, BO Boquerón, CG - Caaguazú, CN - Canindeyú, CZ - Caazapá, CE - Central, CR - Cordillera, GU - Guairá, IT - Itapúa, MI Misiones, NE - Ñeembucú, SP - San Pedro, PA - Paraguarí, $\mathrm{PH}$ - Presidente Hayes.

we haven't been able to place into a province. They are Cerro de Mbatobi, Cerro de Yaguaroux, Perayubi and Cerro León. They have been recorded as PAR.

\section{LIST OF HORNWORTS AND LIVERWORTS RECORDED FOR PARAGUAY}

\section{ANTHOCEROTOPHYTA}

\section{Phaeoceros}

${ }^{* * *}$ P. tenuis (Spruce) Hässel

PAR: SPRUCE $(1888,1890)$ both as Anthoceros tenuis, Stephani (1916) as A. tenuis, Schuster (1992) as A. tenuis. AS: LECTOTYPE of $A$. tenuis, BONNER (1962) as $A$. tenuis, HÄSSEL DE MENÉNDEZ (1986).

\section{Phymatoceros}

*** P. bulbiculosus (Brot.) Stotler, W. T. Doyle \& Crand.-Stotl.

AS: LECTOTYPE of Anthoceros fructuosus, STEPHANI (1897, 1916) both as A. fructuosus, BONNER (1962) as A. fructuosus, HÄSSEL DE MENÉNDEZ (1986) as Phaeoceros fructuosus.

\section{MARCHANTIOPHYTA}

\section{Acanthocoleus}

${ }^{* * *}$ A. aberrans Gradst. var. laevis Gradst.

PAR: Crosby \& Engel (2006). AN: GRADSTEIN (1994).

\section{Archilejeunea}

*** A. auberiana (Mont.) Steph. ex A.Evans

PAR: Dauphin et al. (1998). AN: GRADSTEIN (1994).

${ }^{* * *}$ A. parviflora (Nees) Schiffn.

GU: GRADSTEIN (1994).

\section{Asterella}

${ }^{* * *}$ A. venosa (Lehm. et Lindenb.) A. Evans

PAR: BISCHLER-CAUSSE et al. (2005).

\section{Bryopteris}

${ }^{* * *}$ B. diffusa (Sw.) Nees

PAR: Schäfer-Verwimp \& Pócs (2009). AN: GRADSTEIN (1994).

\section{Calypogeia}

* C. amazonica (Spruce) Steph.

PAR: HUSNOT (1887) as Kantia amazonica, STEPHANI (1908), BISCHLER (1962), Bonner (1963), Fulford (1987).

\section{Caudalejeunea}

${ }^{* * *}$ C. lehmanniana (Gottsche) A. Evans

AN: GRADSTEIN (1994).

\section{Cheilolejeunea}

${ }^{* * *}$ C. clausa (Nees \& Mont.) R. M. Schust.

AN: REINER-DREHWALD (1998). CG: REINERDREHWALD (1998). PA: STEPHANI (1897) as Euosmolejeunea opaca, REINER-DREHWALD (1998).

${ }^{* * *}$ C. rigidula (Nees ex Mont.) R. M. Schust. PAR: Bastos (2012). AN: REINER-DREHWALD (1998).

\section{Chiloscyphus}

${ }^{* * *}$ C. martianus (Nees) J. J. Engel \& R. M. Schust.

PAR: TYPE of Lophocolea paraguayensis, SPRUCE $(1888,1890)$ both as L. paraguayensis, STEPHANI (1906) 
as L. paraguayensis, GEISSLER \& BISCHLER (1985) as L. paraguayensis. CR: STEPHANI (1897) as L. martiana.

\section{Cololejeunea}

${ }^{* * *}$ C. camillii (Lehm.) A. Evans

PAR: Dauphin et al. (2006) as Aphanolejeunea camillii, Alvarenga et al. (2008) as A. camillii, SchäferVerwimp \& Pócs (2009). AN: REINER-DreHWALD (1995a) as A. misionensis.

${ }^{* * *}$ C. cardiocarpa (Mont.) A. Evans

AN: REINER-DREHWALD (1994a).

${ }^{* * *}$ C. clavatopapillata Steph.

PAR: Dauphin et al. (1998) as Aphanolejeunea clavatopapillata, Lüth \& Schäfer-Verwimp (2004) as A. clavatopapillata, Schäfer-Verwimp \& Pócs (2009). AN: REINER-DREHWALD (1995a) as A. clavatopapillata.

${ }^{* * *}$ C. minutissima (Nees \& Mont.) R. M. Schust. subsp. myriocarpa (Nees \& Mont.) R. M. Schust.

PAR: Morales et al. (2008). GU: REINER-DREHWALD (1994a).

${ }^{* * *}$ C. paucifolia (Spruce) Bern.-Lück. \& Pócs

PAR: LECTOTYPE of Lejeunea paucifolia, SPRUCE (1888, 1890) both as L. paucifolia, Stephani (1916) as Physocolea paucifolia, Reiner-Drehwald (1999) as L. paucifolia, Vital \& Visnadi (2000) as Aphanolejeunea paucifolia. PA: GEISSLER \& BISCHLER (1987) as L. paucifolia, REINER-DREHWALD (1995a) as A. paucifolia.

\section{Dumortiera}

${ }^{* * *}$ D. hirsuta (Sw.) Nees

PAR: HUSNOT (1887), SPRUCE (1888), Evans (1919), HÄSSEL DE MENÉNDEZ (1963), Schuster 1992, SchäferVerwimp \& Pócs (2009). AM: BISCHLER-CAUSSE et al. (2005). AN: BISCHLER-CAUSSE et al. (2005). CE: BISCHLER-CAUSSE et al. (2005). CR: STEPHANI (1897). GU: BISCHLER-CAUSSE et al. (2005). PA: BISCHLER-CAUSSE et al. (2005).

\section{Fossombronia}

${ }^{* * *}$ F. porphyrorhiza (Nees) Prosk.

IT: StePhANi (1897) as Noteroclada porphyrorhiza.

\section{Frullania}

${ }^{* *}$ F. cyparioides (Schwägr.) Nees

IT: STEPHANI (1897).

${ }^{* * *}$ F. ericoides (Nees) Mont.

PAR: ORIGINAL MATERIAL of $F$. squarrosa var. $s u b$ julacea, SPRUCE (1888) as F. squarrosa var. subjulacea, Schiffner (1898) as F. squarrosa, Stephani (1910) as F. julacea, Miller et al. (1983) as F. squarrosa. CG: TYPE of $F$. julacea, SPRUCE (1890) as F. julacea, BONNER (1965) as F. julacea, REINER (1988).

${ }^{* * *}$ F. tetraptera Nees \& Mont.

PAR: SPRUCE (1888) as F. conferta, Stephani (1910) as F. conferta. IT: YUZAWA (1991). PA: LECTOTYPE of $F$. conferta, SPRUCE (1890) as $F$. conferta, BONNER (1965) as $F$. conferta, REINER (1988) as F. conferta.

\section{Frullanoides}

${ }^{* * *} F$. liebmaniana (Lindenb. \& Gottsche) van Slageren

AM: GRADSTEIN (1994).

\section{Lejeunea}

${ }^{*}$ L. adpressa Nees

PAR: HUSNOT (1887) as L. drymophila ('drymophylla'), BISCHLER (1966) as L. drymophila.

\section{${ }^{* *}$ L. diaphana Spruce}

PAR: HUSNOT (1887) erroneously as 'Lophocolea'.

*** L. glaucescens Gottsche

PAR: HUSNOT (1887), SPRUCE (1888), BISCHLER (1966).

${ }^{* * *}$ L. grossitexta (Steph.) M. E. Reiner \& Goda

PAR: Holz et al. (2001), Alvarenga et al. (2008), Santos (2008), Schäfer-Verwimp \& Reiner-Drehwald (2009). AN: REINER-DREHWALD (2000), REINERDREHWALD \& GODA (2000).

${ }^{* * *}$ L. laetevirens Nees \& Mont.

PAR: Schäfer-Verwimp \& Pócs (2009). AN: REINERDREHWALD (2000). CG: REINER-DREHWALD (2000) also as L. lepida. PA: REINER-DREHWALD (2000). 
${ }^{* *}$ L. phyllobola Nees \& Mont.

PAR: SPRUCE (1888) as L. cephalandra and L. polycephala, Stephani (1915) as Microlejeunea cephalandra and L. polycephala, BISCHLER (1966) as L. polycephala, Reiner-Drehwald (1999) as L. cephalandra and L. polycephala, Schäfer-Verwimp \& Pócs (2009). AN: REINERDREHWALD (2000). CG: REINER-DREHWALD (2000). CR: LECTOTYPE of L. cephalandra, SPRUCE (1890) as L. cephalandra, GEISSLER \& BISCHLER (1987) as L. cephalandra. PA: LECTOTYPE of L. polycephala, SPRUCE (1890) as L. polycephala, GEISSLER \& BISCHLER (1987) as L. polycephala.

${ }^{* *}$ L. ptosimophylla C.Massal.

PA: REINER-DREHWALD (2000).

${ }^{* * *}$ L. trinitensis Lindenb.

AN: REINER-DREHWALD (2000).

${ }^{*}$ L. trochantha Spruce

PAR: SPRUCE (1888), Stephani (1915), BISCHLER (1966), GEISSLER \& BISCHLER (1987), Reiner-Drehwald (1999). CR: LeCTOTYPE, SPRUCE (1890), GEISSLER \& BISCHLER (1987).

NotE: Lejeunea trochantha is a Cheilolejeunea species of uncertain affinity (Reiner-Drehwald 2006).

\section{Leptolejeunea}

${ }^{* * *}$ L. exocellata (Spruce) A. Evans

AN: REINER-DREHWALD (1995b).

\section{Lophocolea}

${ }^{*}$ L. coadunata (Sw.) Mont.

PAR: SPRUCE (1888). PA: FULFORD (1976).

\section{Marchantia}

${ }^{* * *}$ M. chenopoda L.

AM: BISCHLER-CAUSSE et al. (2005).

${ }^{* * *}$ M. papillata Raddi

PAR: HUSNOT (1887), SPRUCE (1888), STEPHANI (1899a), Svihla (1941), Engel (1990). PA: EVANS (1917), HÄSSEL DE MENÉNDEZ (1963), BISCHLER (1984).

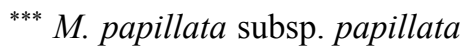

CE: Bischler-Causse et al. (2005). PA: BischlerCausse et al. (2005).

\section{Marchesinia}

${ }^{* * *}$ M. brachiata (Sw.) Schiffn.

PAR: Schäfer-Verwimp \& Pócs (2009). AN: GRADSTEIN (1994).

\section{Mastigolejeunea}

${ }^{* * *}$ M. auriculata (Wilson \& Hook.) Schiffn.

PAR: EVANS (1908), Pagán (1939b), Schuster (1980), Fulford \& Sharp (1990). AM: GRADSTEIN (1994). AN: GRADSTEIN (1994). AS: STEPHANI (1897). CG: REINERDREHWALD (1993).

\section{Metzgeria}

${ }^{* * *}$ M. convoluta Steph.

PAR: Santos (2008). PA: COSTA (2008).

${ }^{* * *}$ M. furcata (L.) Corda

PAR: ORIGINAL MATERIAL of M. planiuscula, SPRUCE (1888) as M. planiuscula, GEISSLER \& BISCHLER (1985) as M. planiuscula.

${ }^{* * *}$ M. myriopoda Lindb.

AN: CosTA (2008).

\section{Microlejeunea}

${ }^{* *}$ M. globosa (Spruce) Steph.

PAR: SPRUCE (1888) as Lejeunea globosa, Stephani (1915), Arnell (1961), GEISSLER \& BISCHLER (1987) as L. globosa, Reiner-Drehwald (1999) as L. globosa, Chuah-Petiot \& Pócs (2003), Lüth \& Schäfer-Verwimp (2004), Morales et al. (2008). PA: LECTOTYPE of L. globosa, SPRUCE (1890) as L. globosa, GEISSLER \& BISCHLER (1987) as L. globosa.

\section{Monoclea}

${ }^{* * *}$ M. gottschei Gradst. \& Mues subsp. elongata Gradst. \& Mues

PA: Bischler-Causse et al. (2005).

\section{Noteroclada}

${ }^{* * *} N$. confluens (Hook.f. \& Taylor) Spruce

PAR: Váňa \& Engel (2013). CR: CRANDALL-STOTLER et al. (2010).

\section{Oxymitra}

${ }^{* * *}$ O. incrassata (Brot.) Sérgio \& Sim-Sim

PAR: HUSNOT (1887) as O. pyramidata, SPRUCE (1888) as O. pyramidata [with a ?], Stephani (1898c) 
as Ruppinia pyramidata, Howe (1914) as Tessellina pyramidata, Casares-Gil (1919) as T. pyramidata, Persson \& Imam (1960) as O. paleacea, Hässel de Menéndez (1963) as O. paleacea, Schuster (1992) as O. paleacea. AS: BISCHLER-CAUSSE et al. (2005). PA: BISCHLERCAUSSE et al. (2005).

\section{Plagiochasma}

${ }^{* * *}$ P. rupestre (J. R. Forst. \& G. Forst.) Steph.

PAR: Bischler (1979), Miller et al. (1983). CR: EVANS (1925)

\section{Plagiochila}

${ }^{* * *}$ P. corrugata (Nees) Nees \& Mont.

PAR: Schäfer-Verwimp \& Pócs (2009), CG: HEINRICHS et al. (2004).

${ }^{* * *}$ P. raddiana Lindenb.

PAR: Schuster (1980) as Plagiochila guilleminiana. PA: STEPHANI (1897) as Plagiochila guilleminiana.

\section{Porella}

${ }^{* * *}$ P. brasiliensis (Raddi) Schiffn.

PAR: Spruce (1888).

${ }^{* * *}$ P. reflexa (Lehm. \& Lindenb.) Trevis.

AN: So (2005).

${ }^{* * *}$ P. swartziana (F. Weber) Trevis.

AN: So (2005).

\section{Radula}

${ }^{* *}$ R. javanica Gottsche

PAR: HUSNOT (1887) as Radula amazonica. AN: REINER-DREHWALD (1994b) as Radula macrostachya.

${ }^{* *}$ R. tectiloba Steph.

PAR: SPRUCE (1888) as $R$. aurantii, Stephani (1910) as R. aurantii, Yamada (1981, 1988, 2000), GEISSLER \& BISCHLER (1990) as R. aurantii, Holz et al. (2001), Schäfer-Verwimp \& Pócs (2009). AN: REINER-DREHWALD (1994b) as $R$. aurantii. AS: LECTOTYPE of $R$. aurantii, SPRUCE (1890) as $R$. aurantii, CASTLE $(1964,1969)$ both as $R$. aurantii, GeISSLER \& BISCHLER (1990) as $R$. aurantii. CG: SYNTYPE of $R$. aurantii, SPRUCE (1890) as $R$. aurantii, CASTLE (1964, 1969) both as $R$. aurantii, GEISSLER \& BISCHLER (1990) as $R$. aurantii, REINER-DREHWALD (1994b) as R. aurantii. CR: CASTLE $(1964,1969)$ both as $R$. aurantii. GU: CASTLE (1964) as $R$. aurantii, REINER-DREHWALD (1994b) as $R$. aurantii. PA: REINER-DREHWALD (1994b) as $R$. aurantii.

\section{Riccardia}

${ }^{* *}$ R. cataractarum (Spruce) Schiffn.

PAR: SPRUCE (1888) as Aneura cataractarum, Stephani (1893) as A. cataractarum, STEPHANI (1899b) as A. cataractarum, Bonner (1962) as A. cataractarum, MeEnKs (1987), Santos (2008), Oliveira \& Bastos (2009), Schäfer-Verwimp \& Pócs (2009). PA: LECTOTYPE of $A$. cataractarum, SPRUCE (1890) as A. cataractarum.

${ }^{* * *}$ R. tenuicula (Spruce) Schiffn. ex Meenks

PAR: HUSNOT (1887) as Aneura 'tenuicaule'. PA: BONNER (1962) also as A. 'tenuicaula'.

\section{Riccia}

${ }^{* * *}$ R. brasiliensis Schiffn.

GU: JOVET-Ast (1991).

${ }^{* * *}$ R. cavernosa Hoffm.

PAR: Bischler-Causse et al. (2005).

NOTE. Bischler-Causse et al. (2005) apparently regard Spruce's (1888) report of Riccia crystallina as referring to this species, although they do not explicitly say so.

${ }^{* * *}$ R. curtisii (Austin) Austin

PAR: STEPHANI (1898b) as Riccia subsimilis. CZ: TYPE of Ricciella subsimilis, STEPHANI (1897) as Ricciella subsimilis, GEISSLER \& BISCHLER (1990) as Ricciella subsimilis, BISCHLER-CAUSSE et al. (2005).

\section{${ }^{* * *} R$. lindmanii Steph.}

AS: TYPE, STEPHANI (1897, 1898a), HÄSSEL DE MENÉNDEZ (1963), GEISSLER \& BISCHLER (1990), JOVETAST (1991), BISCHLER-CAUSSE et al. (2005).

\section{${ }^{* * *} R$. paraguayensis Spruce}

PAR: SPRUCE (1888) as Riccia paraguayensis, GEISSLER \& BISCHLER (1990) as Riccia paraguayensis. AS: LECTOTYPE also of Ricciella macropora, STEPHANI (1897) as Ricciella macropora, STEPHANI (1898b), HÄSSEL DE MENÉNDEZ (1963), GEISSLER \& BISCHLER (1990) also as Ricciella macropora, VIANNA (1992), BISCHLER-CAUSSE et al. (2005). 


\section{${ }^{* * *}$ R. planobiconvexa Steph.}

PAR: Jovet-Ast (1981). CZ: LECTOTYPE of R. tenuilimbata, STEPHANI $(1897,1898 \mathrm{a})$ both as $R$. tenuilimbata, HÄSSEL DE MENÉNDEZ (1963), JOVET-AST (1981, 1991), GEISSLER \& BISCHLER (1990) as R. tenuilimbata, BISCHLER-CAUSSE et al. (2005).

\section{${ }^{* * *}$ R. stenophylla Spruce}

PAR: Spruce (1888), Jovet-Ast (1981), Schuster (1992), Damsholt (2002). AS: TYPE, Spruce (1890), Hässel de Menéndez (1963), Geissler \& Bischler (1990), Bischler-Causse et al. (2005). PH: Bischler-Causse et al. (2005).

\section{${ }^{*}$ R. subtilis (Steph.) Steph.}

PAR: STEPHANI (1898b), GEISSLER \& BISCHLER (1990) as Ricciella subtilis, BISCHLER-CAUSSE et al. (2005). CZ: TYPE of Ricciella subtilis, STEPHANI (1897) as Ricciella subtilis.

NoTE. The type specimen of Riccia subtilis is sterile and Bischler-Causse et al. (2005) did not know where to refer it.

\section{${ }^{* * *}$ R. vitalii Ast}

PAR: JOVET-AST (1991), BISCHLER-CAUSSE et al. (2005).

\section{${ }^{* * *} R$. weinionis Steph.}

PAR: Jovet-Ast (1981) ('Wainionis’).

\section{Schiffneriolejeunea}

${ }^{* * *}$ S. polycarpa (Nees) Gradst.

CR: GRADSTEIN (1994).

\section{Symphyogyna}

\section{${ }^{* * *} S$. aspera Steph. ex F. A. McCormick}

PAR: Pagán (1939), Osakada \& Lisboa (2004), Schäfer-Verwimp \& Pócs (2009). CR: EvANS (1925). PA: URIBE \& AGUIRRE 1995.

\section{${ }^{* * *}$ S. brasiliensis Nees et Mont.}

PAR: SPRUCE (1888).

${ }^{* * *}$ S. sinuata (Sw.) Nees \& Mont.

PAR: HUSNOT (1887), SPRUCE (1888), STEPHANI (1900). CR: STEPHANI (1897).

\section{Taxilejeunea}

${ }^{* * *}$ T. obtusangula (Spruce) A. Evans

PAR: Schäfer-Verwimp \& Pócs (2009.) AN: REINERDREHWALD (2000).

${ }^{* *}$ T. terricola (Spruce) Steph.

PAR: SPRUCE (1888) as Lejeunea terricola, BISCHLER (1966), GEISSLER \& BISCHLER (1987 as L. terricola, 1990), Reiner-Drehwald (1999 as L. terricola, 2000). AS: LECTOTYPE of L. terricola, SPRUCE (1890) as L. terricola, GEISSLER \& BISCHLER (1987) as L. terricola.

DOUBTFUL TAXA

\section{Riccia}

${ }^{* * *} R$. crystallina L.

PAR: SPRUCE (1888), Hässel de Menéndez (1958). This taxon needs confirmation according to BischlerCausse et al. (2005), but they apparently treated the report by Spruce (1888) as Riccia cavernosa.

\section{${ }^{* * *} R$. fluitans L.}

AS: STEPHANI (1897) as Ricciella fluitans. The report was doubted by Bischler-Causse et al. (2005).

\section{SYNONYMS}

Aneura cataractarum Spruce $\equiv$ Riccardia cataractarum

Aneura tenuicula Spruce $\equiv$ Riccardia tenuicula

Anthoceros fructuosus Steph. = Phymatoceros bulbiculosus

Anthoceros tenuis Spruce $\equiv$ Phaeoceros tenuis

Aphanolejeunea camillii (Lehm.) R. M. Schust. $\equiv$ Cololejeunea camillii

Aphanolejeunea clavatopapillata (Steph.) M. E. Reiner $=$ Cololejeunea clavatopapillata

Aphanolejeunea misionensis M. E. Reiner $=$ Cololejeunea camillii

Aphanolejeunea paucifolia (Spruce) M. E. Reiner $\equiv$ Cololejeunea paucifolia

Euosmolejeunea opaca (Gottsche) Steph. = Cheilolejeunea clausa

Frullania conferta Spruce nom. inval. $=$ Frullania tetraptera

Frullania julacea Spruce $=$ Frullania ericoides 
Frullania squarrosa $($ Nees) Nees $=$ Frullania ericoides

Frullania squarrosa var. subjulacea (Mont.) Spruce $=$ Frullania ericoides nica

Kantius amazonicus Spruce $\equiv$ Calypogeia amazo-

Lejeunea cephalandra Spruce $=$ Lejeunea phyllobola

Lejeunea drymophila Spruce $=$ Lejeunea adpressa

Lejeunea globosa Spruce $\equiv$ Microlejeunea globosa

Lejeunea lepida Lindenb. \& Gottsche $=$ Lejeunea laetevirens cifolia

Lejeunea paucifolia Spruce $\equiv$ Cololejeunea pau-

Lejeunea polycephala Spruce nom. inval. $=$ Lejeunea phyllobola cola

Lejeunea terricola Spruce $\equiv$ Taxilejeunea terri-

Lophocolea martiana Nees $\equiv$ Chiloscyphus martianus

Lophocolea paraguayensis Spruce $=$ Chiloscyphus martianus

Metzgeria planiuscula Spruce $=$ Metzgeria furcata

Microlejeunea cephalandra (Spruce) Steph. $=$ Lejeunea phyllobola

Noteroclada porphyrorhiza (Nees) Mitt. $\equiv$ Fossombronia porphyrorhiza

Oxymitra paleacea Bisch. $=$ Oxymitra incrassata

Oxymitra pyramidata (Willd.) Huebener $=$ Oxymitra incrassata

Phaeoceros fructuosus (Steph.) Hässel = Phymatoceros bulbiculosus

Physocolea paucifolia (Spruce) Steph. $\equiv$ Cololejeunea paucifolia

Plagiochila guilleminiana Mont. ex Lindenb. $=$ Plagiochila raddiana

Radula amazonica Spruce $=$ Radula javanica

Radula aurantii Spruce $=$ Radula tectiloba

Radula macrostachya Lindenb. \& Gottsche $=R a$ dula javanica

Riccia subsimilis (Steph.) Steph. $=$ Riccia curtisii

Riccia tenuilimbata Steph. = Riccia planobiconvexa

Ricciella fluitans (L.) A. Braun $\equiv$ Riccia fluitans

Ricciella macropora Steph. = Riccia paraguayensis

Ricciella subsimilis Steph. $=$ Riccia curtisii
Ricciella subtilis Steph. $\equiv$ Riccia subtilis

Ruppinia pyramidata (Willd.) Corda nom. illeg. = Oxymitra incrassata

Tessellina pyramidata (Willd.) Dumort. $=$ Oxymitra incrassata

ACKNOWLEDGEMENTS. The Early Land Plants Today project (ELPT) has been generously supported in part by the Global Biological Information Facility (GBIF) Seed Money Award No. 2007-41, activities facilitated in part by funding from the Biodiversity Synthesis Center of the Encyclopedia of Life (BioSynC), partial funding from the National Science Foundation (Award No's 0749762, 1115002), the Warwick Foundation, and the Negaunee Foundation.

\section{REFERENCES}

Alvarenga L. D. P., do ParÁ Marques de Oliveira J. R., Pereira Silva M. P., Costa S. O. \& Pôrto K. C. 2008. Liverworts of Alagoas State, Brazil. Acta Bot. Brasilica 22: $878-890$.

ARNELL S. 1961. A new species of Bazzania from Chile. Svensk Bot. Tidskr. 55: 394.

BAstos C. J. P. 2012. New combinations and synonyms in Cheilolejeunea (Spruce) Schiffn. (Lejeuneaceae, Marchantiophyta). J. Bryol. 34: 312-315.

Bischler H. 1962. The genus Calypogeja Raddi in Central and South America III. Subgenus Calypogeja, subgroups 4 and 5. Candollea 18: 95-128.

BISCHLER H. 1966. Recherche sur l'anatomie de la tige chez les Lejeuneaceae III. Les sous-familles Lejeuneoideae, tribu Lejeuneeae, et Tuyamaelloideae. Rev. Bryol. Lichénol. 34: 601-675.

BISCHLER H. 1979. Plagiochasma Lehm. et Lindenb. III. Les taxa d'Asie et d'Océanie. J. Hattori Bot. Lab. 45: 25-79.

BisCHLER H. 1984. Marchantia L., the New World species. Bryophyt. Biblioth. 26: 1-228.

Bischler-CAusse H., GRADSTEIN S. R., JOVET-Ast S., LONG D. G. \& Salazar Allen N. 2005. Marchantiidae. Fl. Neotrop. Monogr. 97: 1-267.

Bonner C. E. B. 1962. Index Hepaticarum. Pars II. Achiton to Balantiopsis. J. Cramer, Weinheim.

Bonner C. E. B. 1963. Index Hepaticarum. Pars III. Barbilophozia to Ceranthus. J. Cramer, Weinheim.

BonNer C. E. B. 1965. Index Hepaticarum. Pars V. Delavayella to Geothallus. J. Cramer, Weinheim.

CASAREs-Gil A. 1919. Flora Ibérica Briofitas. Part 1, Hepaticas. Museo Nacional de Ciencias Naturales, Madrid. 
CAstle H. 1964. A revision of the genus Radula. Part II. Subgenus Acroradula. Section 8. Acutilobulae. Rev. Bryol. Lichénol. 33: 185-210.

CAstle H. ['1968'] 1969. Radula (L.) Dumortier. - A synopsis of the taxonomic revision of the genus. Rev. Bryol. Lichénol. 36: 5-44.

Catterson T. M. \& Fragano F. V. 2004. Tropical forestry and biodiversity conservation in Paraguay. Final Report of a Section 118/119 Assessment, EPIQ II Task No. 1, USAID, Asuncion, Paraguay.

ChuAh-Petiot M. S. \& Pócs T. 2003. East African bryophytes XIX. Acta Bot. Hung. 45: 53-64.

Costa D. P. 2008. Metzgeriaceae. Flora Neotropica 102: $1-169$.

Crandall-Stotler B., Stotler R. E., Zhang L., Forrest L. L. 2010. On the morphology, systematics and phylogeny of Noteroclada (Noterocladaceae, Marchantiophyta). Nova Hedwigia 91: 421-450.

Crosby M. R. \& Engel J. J. 2006. Index of Hepatics 1974 2000. Hattori Botanical Laboratory, Nichinan.

DAMSHOLT K. 2002. Illustrated flora of Nordic liverworts and hornworts. Nordic Bryological Society, Lund.

Dauphin G., Gradstein S.R., Bernecker-Lücking A. \& MoRALES M. I. 1998. Additions to the hepatic flora of Costa Rica II. Lindbergia 23: 74-80.

Dauphin G., Pócs T., Villarreal J. C. \& Salazar Allen N. 2006. Nuevos registros de hepáticas y anthocerotófitas para Panamá. Trop. Bryol. 27: 73-85.

ENGEL J. J. 1990. Falkland Islands (Islas Malvinas) Hepaticae and Anthocerotophyta: A taxonomic and phytogeographic study. Fieldiana, Bot., n.s. 25: 1-209.

Evans A. W. 1908. Hepaticae of Puerto Rico. VIII. Symbiezidium, Marchesinia, Mastigolejeunea, Caudalejeunea, and Bryopteris. Bull. Torrey Bot. Club 34: 533-568.

Evans A. W. 1917. The American species of Marchantia. Trans. Connecticut Acad. Arts 21: 253-261.

Evans A. W. 1919. A taxonomic study of Dumortiera. Bull. Torrey Bot. Club 46: 167-182.

Evans A. W. 1925. The lobate species of Symphyogyna. Trans. Connecticut Acad. Arts 27: 1-50.

Fourier B. 2005. Les découvertes de Balansa se sont étendues jusqu'aux antipodes. Bull. Assoc. Parcs Bot. France 40: $2-9$.

FULFORD M. 1976. Manual of the leafy Hepaticae of Latin America IV. Mem. New York Bot. Gard. 11: 395-535.

FULFORD M. 1987. Leafy hepaticae of Dominica: additions to the Hepaticae Elliottianae by Richard Spruce in 1895 . Mem. New York Bot. Gard. 45: 264-278.

FUlford M. \& SHARP A. J. 1990. The leafy Hepaticae of
Mexico: one hundred and twenty-seven years after C. M. Gottsche. Mem. New York Bot. Gard. 63: 1-86.

GeISSLER P. \& BisCHLER H. 1985. Index Hepaticarum. 10. Lembidium to Mytilopsis. J. Cramer, Berlin.

Geissler P. \& BischleR H. 1987. Index Hepaticarum. 8/9. Jungermannia to Lejeunites. J. Cramer, Berlin.

GeIsSler P. \& BischleR H. 1990. Index Hepaticarum. 12. Racemigemma to Zoopsis. J. Cramer, Berlin.

GradsteIn S. R. 1994. Lejeuneaceae: Ptychantheae, Brachiolejeuneae. Fl. Neotrop. Monogr. 62: 1-217.

HÄSSEL DE MENÉNDEZ G. 1958. Nota preliminar sobre especies de Riccia halladas en Argentina. Bol. Soc. Argent. Bot. 7: 99-115.

HÄSSEL DE MENÉNDEZ G. ['1962'] 1963. Estudio de las Anthocerotales y Marchantiales de la Argentina. Opera Lilloana 7: 1-298.

HÄSSEL DE MENÉNDEZ G. 1986. Neue Lebermoosfunde aus dem Nordosten Argentiniens - New findings of hepaticae from northeastern Argentina - Hallazgos nuevos de hepáticas en el nordeste de la Argentina. Veröff. Geobot. Inst. ETH Stiftung Rübel Zürich 91: 293-304.

Heinrichs J., Groth H., Lindner M., Renker C., Pócs T. \& PRÖSCHOLD T. 2004. Intercontinental distribution of Plagiochila corrugata (Plagiochilaceae, Hepaticae) inferred from nrDNA ITS sequences and morphology. J. Linn. Soc., Bot. 146: 469-481.

Holz I., HeINRICHS J., SCHÄFER-VERWIMP A. \& GRADSTEIN S. R. 2001. Additions to the hepatic flora of Costa Rica III. Cryptog. Bryol. 22: 255-273.

Howe M. A. 1914. Oxymitra (Tessellina) in the United States. Bryologist 17: 72-75.

Huang C., Kim S., Song K., Townsend J. R. G., Davis P., Altstatt A., Rodas O., YANOSKy A., Clay R., TUCKer C. J. \& MUSINSKY J. 2009. Assessment of Paraguay's forest cover change using Landsat observations. Global and Planetary Change 67: 1-12.

Husnot T. 1887. Musci Exotici. Rev. Bryol. 14: 56-58.

Jovet-Ast S. 1981. Riccia d'Amérique tropicale. Occas. Pap. Farlow Herb. Cryptog. Bot. 16: 111-115.

Jovet-Ast S. 1991. Riccia (Hépatiques, Marchantiales) d'Amérique latine. Taxons du sous-genre Riccia. Cryptog. Bryol. Lichénol. 12: 189-370.

LÜTH M. \& SCHÄFER-VERWIMP A. 2004. Additions to the bryophyte flora of the Neotropics. Trop. Bryol. 25: 7-17.

MeEnKS J. L. D. 1987. Studies on Colombian cryptogams XXVIII. A guide to the tropical Andean species of Riccardia (Hepaticae). J. Hattori Bot. Lab. 62: 161-182.

Miller H. A., Whittier H. O. \& Whittier B. A. 1983. Prodromus Florae Hepaticarum Polynesiae. Bryophyt. Biblioth. 25: 1-423. 
Morales T., Moreno E. \& García M. 2008. Briofitas del área recreativa del Jardin Botánico de Caracas. Ernstia 18: $37-58$.

MYERS N. 1988. Threatened biotas: "Hot spots" in tropical forests. The Environmentalist 8: 1-20.

Oliveira H. C. \& BAstos C. J. P. 2009. Antóceros (Anthocerotophyta) e hepáticas talosas (Marchantiophyta) da Chapada da Ibiapaba, Ceará, Brasil. Rodriguésia 60: 477-484.

Olson D. M. \& DinERSTEIN E. 2002. The Global 200: priority ecoregions for global conservation. Ann. Missouri Bot. Gard. 89: 199-224.

Olson D. M., Dinerstein E., Wikramanayake E. D., Burgess N. D., Powell G. V. N., Underwood E. C., D’Amico J. A., Itoua I., Strand H. E., Morrison J. C., Loucks C. J., AllnutT T. F., Ricketts T. H., KuRA Y., LAmoreuX J. F., Wettengel W. W., Hedao P. \& Kassem K. R. 2001. Terrestrial ecoregions of the world: a new map of life on Earth. Bioscience 51: 933-938.

OSAKADA A. \& LisBoA R. C. L. 2004. Novas ocorrências de hepáticas (Marchantiophyta) para o estado do Pará, Brasil. Acta Amazonica 34: 197-200.

O’Shea B. J. \& Price M. 2008. An updated checklist of the mosses of Paraguay. Trop. Bryol. 29: 6-37.

PAGÁN F. M. 1939a. A preliminary list of the hepaticae of Puerto Rico including Vieques and Mona Island. Bryologist 42: 1-12.

PAGÁN F. M. 1939b. A preliminary list of the hepaticae of Puerto Rico including Vieques and Mona Island (concluded). Bryologist 42: 71-82.

Persson H. \& IMAM M. 1960. The first find of a Riella in Egypt and some words about the distribution of the genus in the world. Rev. Bryol. Lichénol. 29: 1-9.

REINER M. E. 1988. Contribución al conocimiento de las hepaticas del noreste de la Provincia de Buenos Aires (Argentina). Frullaniaceae (Jungermanniales). Bol. Soc. Argent. Bot. 25: 301-325.

REINER-DREHWALD M. E. 1993. Las Lejeuneaceae (Hepaticae) de Misiones, Argentina I. Las especies holostipas. Trop. Bryol. 7: 13-45.

REINER-DREHWALD M. E. 1994a. Las Lejeuneaceae (Hepaticae) de Misiones, Argentina II. Cololejeunea. Trop. Bryol. 9: $79-88$.

REINER-DREHWALD M. E. 1994b. El género Radula (Radulaceae, Hepaticae) en el Noreste de Argentina. Trop. Bryol. 9: 5-22.

REINER-DREHWALD M. E. 1995a. Las Lejeuneaceae (Hepaticae) de Misiones, Argentina. IV. Aphanolejeunea. Trop. Bryol. 10: 29-40.

REINER-DREHWALD M. E. 1995b. Las Lejeuneaceae (Hepaticae) de Misiones, Argentina III. Drepanolejeunea y Leptolejeunea. Trop. Bryol. 10: 21-27.
REINER-DREHWALD M. E. 1998. Las Lejeuneaceae (Hepaticae) de Misiones, Argentina V. Cheilolejeunea y Lepidolejeunea. Trop. Bryol. 14: 53-68.

REINER-DREhWALd M. E. 1999. Catalogue of the genus Lejeunea Lib. (Hepaticae) of Latin America. Bryophyt. Biblioth. 54: 1-101.

REINER-DREHWALD M. E. 2000. Las Lejeuneaceae (Hepaticae) de Misiones, Argentina VI. Lejeunea y Taxilejeunea. Trop. Bryol. 19: 81-131.

REINER-DREHWALD M. E. 2006. Type studies on neotropical Lejeuneaceae (Jungermanniopsida). Cheilolejeunea and Lepidolejeunea. Nova Hedwigia 83: 473-482.

REINER-DREHWALD M. E. \& GODA A. 2000. Revision of the genus Crossotolejeunea (Lejeuneaceae, Hepaticae). J. Hattori Bot. Lab. 89: 1-54.

SAntos N. D. 2008. Hepáticas da Mata Atlântica do estado do Rio de Janeiro: diversidade, fitogeografia e conservação. Instituto de Pesquisas Jardim Botânico do Rio Janeiro, Escola Nacional de Botânica Tropical, Rio de Janeiro, $143 \mathrm{pp}$.

SCHÄFER-VERWIMP A. \& PóCS T. 2009. Contributions to the hepatic flora of the Dominican Republic, West Indies. Acta Bot. Hung. 51: 367-425.

SCHÄFER-VERWIMP A. \& REINER-DREHWALD E. 2009. Some additions to the bryophyte flora of Guadeloupe, West Indies, and new synonyms in the genera Diplasiolejeunea and Lejeunea (Lejeuneaceae). Cryptog. Bryol. 30: 357-375.

SCHIFFNER V. 1898. Conspectus Hepaticarum Archipelagi Indici. Staatsdruckerei, Batavia.

Schuster R. M. 1980. The Hepaticae and Anthocerotae of North America. 4. Columbia University Press, New York.

SCHUSTER R. M. 1992. The Hepaticae and Anthocerotae of North America. 6. Columbia University Press, New York.

So M. L. 2005. Porella (Porellaceae, Marchantiophyta) in Latin America. New Zealand J. Bot. 43: 301-321.

SÖDERSTRÖM L, HAGBorg A, von KonRAT M, RENNER MAM. 2008. Early Land Plants Today: Liverwort checklist of checklists. Fieldiana, Bot., n.s. 47: 105-130.

SÖDERStröm L., Gradstein S. R. \& Hagborg A. 2010. Checklist of hornworts and liverworts for Java. Phytotaxa 9: 53-149.

SPRUCE R. 1888. Hepaticae paraguayensis, Balansa lectae. Rev. Bryol. 15: 34-35.

SPRUCE R. ['1889'] 1890. Hepaticae novae Americanae tropicae et aliae. Bull. Soc. Bot. France 36: CLXXXIX-CCVII.

StePhAni F. 1893. Hepaticarum species novae. Pars II. Hedwigia 32: 137-147.

STEPHANI F. 1897. Die Lebermoose der ersten Regnell'schen 
expedition nach Südamerika. Bih. Kongl. Svenska Vetensk.Akad. Handl. 23(2): 1-36.

StePHANi F. 1898a. Species Hepaticarum 1. Bull. Herb. Boissier 6(4): 309-343.

StePHANI F. 1898b. Species Hepaticarum 1. Bull. Herb. Boissier 6(5): 361-378.

StePHANi F. 1898c. Species Hepaticarum 1. Bull. Herb. Boissier 6(10): 757-799.

StePHANi F. 1899a. Species Hepaticarum 1. Bull. Herb. Boissier 7(5): 381-407.

StePHANI F. 1899b. Species Hepaticarum 1. Bull. Herb. Boissier 7(10): 727-764.

StePhani F. 1900. Species Hepaticarum 1. Mém. Herb. Boissier 11: 1-49.

Stephani F. 1906. Species Hepaticarum 3. Bull. Herb. Boissier sér. 2 6(11): 935-966.

StePhani F. 1908. Species Hepaticarum 3. Bull. Herb. Boissier sér. 2 8(9): 661-696.

Stephani F. 1910. Species Hepaticarum 4. George \& Cie, Genève \& Bale.

Stephani F. 1915. Species Hepaticarum 5. George \& Cie, Genève \& Bale.

Stephani F. 1916. Species Hepaticarum 5. George \& Cie, Genève \& Bale.

SVIHLA R. D. 1941. The status of Marchantia domingensis. Bryologist 44: 46-48.

Tabarelli M., Pinto L. P., Silva J. M. C., Hirota M. \& BedÊ L. 2005. Challenges and opportunities for biodiversity conservation in the Brazilian Atlantic Forest. Conservation Biology 19: 695-700.

URIBE J. \& AGUIRRE J. 1995. Las especies colombianas del género Symphyogyna (Hepaticae: Pallaviciniaceae). Caldasia 17: 429-457.

VÁŇA J. \& ENGEL J. J. 2013. The liverworts and hornworts of the Tristan da Cunha group. Mem. New York Bot. Gard. 105: $1-138$.

ViAnNA E. C. 1992. Esporos de Riccia paraguayensis Spruce. Iheringia, Bot. 42: 109-111.

Vital D. M. \& Visnadi S. R. 2000. New records and notes on Brazilian bryophytes. J. Hattori Bot. Lab. 88: 191-197.

Von KonRat M., SöDERSTRÖM L. \& HAgBORG A. 2010. The Early Land Plants Today project: A community-driven effort and a new partnership with Phytotaxa. Phytotaxa 9: 11-21.

YAMADA K. 1981. Notes on the type specimens of Radula taxa from Latin America (2). J. Hattori Bot. Lab. 49: 385-398.

YAMADA K. 1988. The genus Radula from Cuba. J. Hattori Bot. Lab. 65: 379-390.

YAMADA K. 2000. A collection of the genus Radula (Radulaceae; Hepaticae) made by Marko Lewis in Bolivia. J. Hattori Bot. Lab. 88: 257-265.

YuZAWA Y. 1991. A monograph of subgen. Chonanthelia of gen. Frullania (Hepaticae) of the world. J. Hattori Bot. Lab. 70: 181-291.

ZARDINI E. M. 1993. Paraguay's floristic inventory. Research \& Exploration 9, 128-131.

Received 1 May 2013 